\title{
Noninvasive Assessment of IDH Mutational Status in World Health Organization Grade II and III Astrocytomas Using DWI and DSC-PWI Combined with Conventional MR Imaging
}

\author{
(D) Z. Xing, (D) X. Yang, (DD. She, (D) Y. Lin, DY. Zhang, and (DD. Cao
}

\begin{abstract}
BACKGROUND AND PURPOSE: Isocitrate dehydrogenase (IDH) has been shown to have both diagnostic and prognostic implications in gliomas. The purpose of this study was to examine whether DWI and DSC-PWI combined with conventional MR imaging could noninvasively predict IDH mutational status in World Health Organization grade II and III astrocytomas.
\end{abstract}

MATERIALS AND METHODS: We retrospectively reviewed DWI, DSC-PWI, and conventional MR imaging in 42 patients with World Health Organization grade II and III astrocytomas. Minimum ADC, relative ADC, and relative maximum CBV values were compared between $I D H$-mutant and wild-type tumors by using the Mann-Whitney $U$ test. Receiver operating characteristic curve and logistic regression were used to assess their diagnostic performances.

RESULTS: Minimum ADC and relative ADC were significantly higher in IDH-mutated grade II and III astrocytomas than in IDH wild-type tumors $(P<.05)$. Minimum ADC with the cutoff value of $\geq 1.01 \times 10^{-3} \mathrm{~mm}^{2} / \mathrm{s}$ could differentiate the mutational status with a sensitivity, specificity, positive predictive value, and negative predictive value of $76.9 \%, 82.6 \%, 91.2 \%$, and $60.5 \%$, respectively. The threshold value of $<2.35$ for relative maximum CBV in the prediction of $I D H$ mutation provided a sensitivity, specificity, positive predictive value, and negative predictive value of $100.0 \%, 60.9 \%, 85.6 \%$, and $100.0 \%$, respectively. A combination of DWI, DSC-PWI, and conventional MR imaging for the identification of IDH mutations resulted in a sensitivity, specificity, positive predictive value, and negative predictive value of $92.3 \%$, $91.3 \%, 96.1 \%$, and $83.6 \%$.

CONCLUSIONS: A combination of conventional MR imaging, DWI, and DSC-PWI techniques produces a high sensitivity, specificity, positive predictive value, and negative predictive value for predicting IDH mutations in grade II and III astrocytomas. The strategy of using advanced, semiquantitative MR imaging techniques may provide an important, noninvasive, surrogate marker that should be studied further in larger, prospective trials.

ABBREVIATIONS: $A D C_{\text {min }}=$ minimum $A D C ; C M R I=$ conventional MR imaging; $I D H=$ isocitrate dehydrogenase; $r A D C=$ relative $A D C ; r C B V=$ relative $C B V$; $\mathrm{rCBV}_{\max }=$ relative maximum $\mathrm{CBV} ; \mathrm{WHO}=$ World Health Organization

nfiltrating astrocytomas are the most common primary central nervous system tumors, ranging variably from grade II to IV according to the 2007 World Health Organization classification

Received October 22, 2016; accepted after revision February 6, 2017.

From the Department of Radiology, First Affiliated Hospital of Fujian Medical University, Fuzhou, P.R. China.

Zhen Xing and Xiefeng Yang contributed equally to this work.

This work was supported by the Leading Project of the Department of Science and Technology of Fujian Province (No. 2016Y0101) to Dairong Cao, Special Funds of the Provincial Finance of Fujian Province (No. BPB-CDR2013) to Dairong Cao, and the Research Foundation for Young Scholars of the Health Department of Fujian Province (No. 2013-1-34) to Zhen Xing.

Please address correspondence to Dairong Cao, MD, Department of Radiology, First Affiliated Hospital of Fujian Medical University, 20 Cha-Zhong Rd, Fuzhou 350005, Fujian, P.R. China; e-mail: dairongcao@163.com

- Indicates open access to non-subscribers at www.ajnr.org

http://dx.doi.org/10.3174/ajnr.A5171 system. ${ }^{1,2}$ Glioma grading is based on histopathologic analysis of tumor differentiation, mitotic activity, cellularity, nuclear atypia, and the extent of microvascular proliferation and may result in a great deal of interobserver variability. ${ }^{1-3}$ Therefore, quantitative molecular analyses have the potential to reduce subjectivity and improve diagnosis, prognostication, risk stratification, and management plans. Notably, in the 2016 World Health Organization (WHO) classification, grade II and III astrocytomas are molecularly divided into isocitrate dehydrogenase (IDH) mutant, IDH wild type, and not otherwise specified categories, emphasizing the value of $I D H$ mutation status in astrocytomas. ${ }^{4}$

IDH gene mutations, originally discovered in high-grade gliomas in 2008 , exist in $60 \%-90 \%$ of grade II and III astrocytomas. ${ }^{5,6}$ The IDH gene (including IDH1 and IDH2 genes) plays prominent roles in the metabolism, pathogenesis, and progression of astrocytomas. ${ }^{7-9}$ In addition, stratification of grade II and III gliomas 
into subsets defined by IDH mutation would help identify subgroups with distinct prognostic characteristics, therapeutic response, and clinical management. ${ }^{10-16}$ In a study with a cohort of 475 patients, comparison of overall survival between those with WHO grade II and III IDH-mutated astrocytomas showed no remarkable difference, whereas the patients with $I D H$-mutated tumors survived much longer than those with $I D H$ wild-type tumors. ${ }^{10}$ Patients with grade II astrocytomas without $I D H$ mutation were shown to have a poorer prognosis with a 5-year progression-free survival and overall survival rate of $14 \%$ and $51 \%$, respectively, compared with $42 \%$ and $93 \%$ for those with $I D H$ mutant tumors $(P<.001) .{ }^{17}$ Moreover, patients with gliomas whose lesions had IDH mutations were more sensitive to chemoradiation therapy and survived longer than those with wild-type IDH. ${ }^{10,15}$

Currently, immunohistochemical staining and DNA sequencing are the most common methods for determining the $\mathrm{IDH}$ mutational status in gliomas. IDH gene mutations may reflect alterations in metabolism, cellularity, and angiogenesis, which may manifest characteristic features on DWI and DSC-PWI. ${ }^{8,18}$ DWI can noninvasively provide direct insight into the microscopic physical properties of tissues through observing the Brownian movement of water and reflecting cellularity within the lesions by ADC values. ${ }^{19-21}$ In vivo measurement of relative CBV (rCBV) has been demonstrated to correlate with tumor vascularity. ${ }^{22-24}$ ADC values derived from DWI and DSC-PWI have been used to detect IDH gene status in gliomas in recent research. ${ }^{24-26}$ Meanwhile, conventional MR imaging (cMRI) was also used to assess other characteristics of gliomas (eg, location, distinctness of borders, enhancement, and edema). ${ }^{17,27,28}$

To our knowledge, there is no study in the literature combining cMRI with diffusion and perfusion techniques to distinguish IDH genotypes. The purpose of this study was to explore whether a novel approach, in which DWI and DSC-PWI were combined with cMRI, was able to noninvasively predict $I D H$ mutational status in WHO grade II and III astrocytomas.

\section{MATERIALS AND METHODS Patients}

The ethics committee of our hospital approved this retrospective study, and the requirement for patient informed consent was waived due to the nature of the retrospective study. Ninety-six patients who underwent surgical resection or stereotactic biopsy at our institution from July 2014 through June 2016 were selected. The inclusion criteria were as follows: 1) definite histopathologic diagnosis of grade II and III astrocytomas based on the WHO 2016 classification criteria, 2) cMRI, DWI, and DSC-PWI performed before treatment, and 3) all data available in 3T MR imaging. As a result, 42 patients (26 males and 16 females; mean age, $41.83 \pm 15.98$ years; age range, $8-72$ years) were included in the study.

\section{MR Imaging Techniques}

Images were acquired in the routine clinical work-up on a 3T MR imaging system (Magnetom Verio Tim; Siemens, Erlangen, Germany) with an 8-channel head matrix coil. The conventional MR imaging protocols consisted of the following sequences: axial T1-
Table 1: The main clinical and CMRI features of IDH mutational status in grade II and III astrocytomas ${ }^{\mathrm{a}}$

\begin{tabular}{|c|c|c|c|}
\hline & $\begin{array}{c}\text { IDH } \\
\text { Mutation } \\
(n=17)\end{array}$ & $\begin{array}{c}\text { IDH Wild } \\
\text { Type } \\
(n=25)\end{array}$ & $P$ Value \\
\hline Sex (male/female) & $9 / 8$ & $17 / 8$ & .157 \\
\hline Age (yr) & $35.76 \pm 9.13$ & $45.96 \pm 18.36$ & .041 \\
\hline Location & & & .006 \\
\hline Frontal lobe & $9(52.9 \%)$ & $4(16.0 \%)$ & \\
\hline Parietal lobe & $1(5.9 \%)$ & $3(12.0 \%)$ & \\
\hline Temporal lobe & $6(35.3 \%)$ & $4(16.0 \%)$ & \\
\hline Occipital lobe & 0 & 0 & \\
\hline Insular lobe & $1(5.9 \%)$ & $3(12.0 \%)$ & \\
\hline Others & 0 & 11 (44.0\%) & \\
\hline Homogeneity & & & .439 \\
\hline Homogeneous & $4(23.5 \%)$ & $7(28.0 \%)$ & \\
\hline Heterogeneous & $13(76.5 \%)$ & $18(72.0 \%)$ & \\
\hline Edema & & & .746 \\
\hline Presence & $3(17.6 \%)$ & $7(28.0 \%)$ & \\
\hline Absence & $14(82.4 \%)$ & 18 (72.0\%) & \\
\hline Borders & & & .037 \\
\hline Sharp & 11 (64.7\%) & $8(32.0 \%)$ & \\
\hline Indistinct & $6(35.3 \%)$ & $17(68.0 \%)$ & \\
\hline Contrast enhancement & & & .286 \\
\hline No & 11 (64.7\%) & $12(48.0 \%)$ & \\
\hline Yes & $6(35.3 \%)$ & $13(54.0 \%)$ & \\
\hline Histology & & & .051 \\
\hline Grade II astrocytomas & $12(70.6 \%)$ & $12(48.0 \%)$ & \\
\hline Grade III astrocytomas & $5(29.4 \%)$ & $13(54.0 \%)$ & \\
\hline
\end{tabular}

${ }^{a}$ Data are number (\%) unless otherwise indicated.

weighted gradient-echo imaging $(\mathrm{TR}=250 \mathrm{~ms}$; $\mathrm{TE}=2.48 \mathrm{~ms})$, axial $\mathrm{T} 2$-weighted turbo spin-echo imaging $(\mathrm{TR}=4000 \mathrm{~ms}$; $\mathrm{TE}=$ $96 \mathrm{~ms}$ ), axial fluid-attenuated inversion recovery imaging (TR/ $\mathrm{TE}=9000 / 94 \mathrm{~ms} ; \mathrm{TI}=2500 \mathrm{~ms}$ ), and 3 orthogonal plane contrast-enhanced gradient-echo T1-weighted imaging scans (TR/ TE, 250/2.48 ms) acquired following the acquisition of DSC-PWI sequences. The section thickness $(5 \mathrm{~mm})$, intersection gap (1 $\mathrm{mm})$, and FOV $(220 \times 220 \mathrm{~mm})$ were uniform in all sequences.

DWI was performed in the axial plane with a spin-echo echoplanar sequence before injection of contrast material. The imaging parameters used were as follows: TR/TE $=8200 / 102 \mathrm{~ms}$, $\mathrm{NEX}=2.0$, section thickness $=5 \mathrm{~mm}$, intersection gap $=1 \mathrm{~mm}$, FOV $=220 \times 220 \mathrm{~mm}$. The b-values were 0 and $1000 \mathrm{~s} / \mathrm{mm}^{2}$ with diffusion gradients encoded in the 3 orthogonal directions to generate 3 sets of diffusion-weighted images. Processing of the ADC map was generated automatically by the MR imaging system.

DSC-PWI was performed with a gradient-recalled $\mathrm{T}^{*}$ weighted echo-planar imaging sequence. The imaging parameters were as follows: TR/TE $=1000-1250 / 54 \mathrm{~ms}$, flip angle $=35^{\circ}$, section thickness $=5 \mathrm{~mm}$, intersection gap $=1 \mathrm{~mm}, \mathrm{NEX}=1.0$, FOV $=220 \times 220 \mathrm{~mm}$. During the first 3 phases, images were acquired before injecting the contrast material to establish a precontrast baseline. When the scan was to the fourth phase of DSCPWI, a bolus of gadobenate dimeglumine at a dose of 0.1 $\mathrm{mmol} / \mathrm{kg}$ of body weight and $5 \mathrm{~mL} / \mathrm{s}$ was injected intravenously with an MR imaging-compatible power injector. After we injected a bolus of the contrast material, a 20.0-mL bolus of saline was administered at the same injection rate. The series of $20 \mathrm{sec}-$ tions, 60 phases, and 1200 images was obtained in 1 minute 36 seconds.

AJNR Am J Neuroradiol 38:1138-44 Jun 2017 www.ajnr.org 1139 

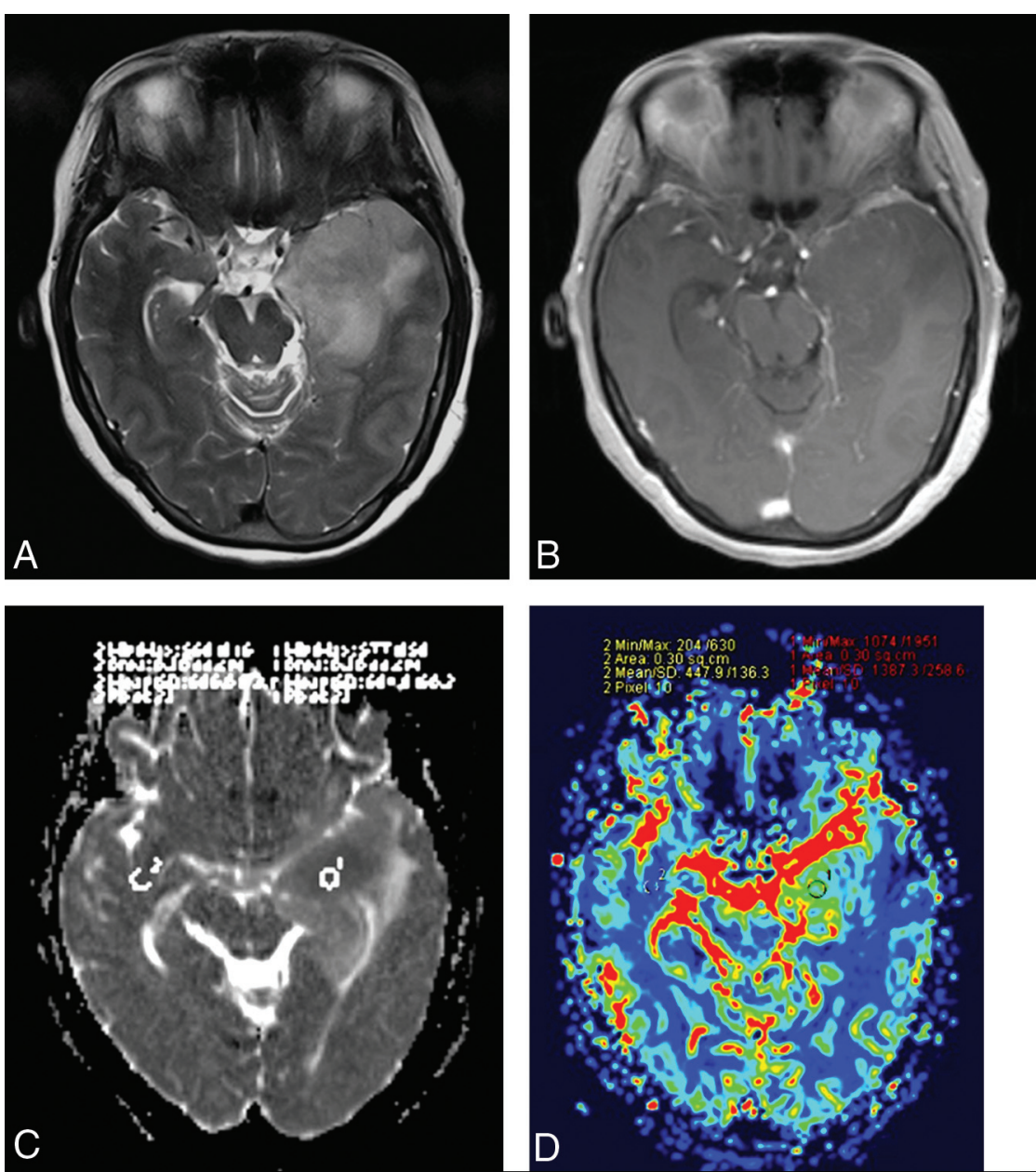

FIG 1. A 52-year-old woman with a diffuse astrocytoma without IDH mutation. A, Axial T2WI demonstrates heterogeneous high signal intensity with indistinct borders on the left temporal lobe. $B$, Contrast-enhanced axial T1-weighted image demonstrates a lesion enhancement with blurred borders. $C, A$ corresponding ADC map shows the tumor with a decreased ADC value $\left(A D C_{\min }=0.684 \times 10^{-3} \mathrm{~mm}^{2} / \mathrm{s}, \mathrm{rADC}=1.08\right) . D$, Correlative color $\mathrm{CBV}$ image shows elevated perfusion with the calculated $\mathrm{rCBV}_{\max }$ of 3.10 .

\section{Data Processing}

Image postprocessing of perfusion data and perfusion measurements was performed on an off-line syngo B19 workstation (Siemens) with standard software. All cMRI data with respect to tumor locations, heterogeneity, borders, peritumoral edema, and contrast-enhancement pattern were assessed by 2 neuroradiologists who were blinded to tumor histology and molecular characteristics. Tumor location, considered to be the lobe within which the bulk of the tumor resided, was divided into 6 groups: frontal lobe, temporal lobe, parietal lobe, insular lobe, occipital lobe, and others (basal ganglia, thalamus, brain stem, and cerebellum). Edema was defined as a nonenhanced area on contrast-enhanced T1WI and higher signal outside the tumoral solid area on T2WI and FLAIR. Tumor borders were classified as sharp or indistinct on the basis of T2WI and FLAIR sequences (relatively decreased signal intensity on T2WI or FLAIR should be regarded as tumor area rather than edema in gliomas). A senior neuroradiologist made the final decision when 2 observers disagreed.

For evaluation of DWI data, ADC values were measured by manually selecting ROIs inside the tumor regions on the ADC maps. All continuous sections including tumors were observed.
At least 5 small round ROIs (30-40 $\mathrm{mm}^{2}$ ) were placed inside the tumors on the ADC maps without overlap. The bigger the tumor was, the more ROIs were selected. Finally, the ROI with the lowest $\mathrm{ADC}$ value was chosen to calculate minimum $\mathrm{ADC}\left(\mathrm{ADC}_{\text {min }}\right)$. We made the ROI placement from the solid portion of the lesion (defined on T2WI and contrast-enhanced T1WI), avoiding hemorrhagic, cystic, necrotic, or apparent blood vessel regions that might influence the ADC values. The minimum $\mathrm{ADC}$ is calculated as the mean value of the ROI of the lowest ADC value. The same method was applied to a corresponding area in the contralateral normal-appearing white matter judged on both T2WI and contrast-enhanced T1WI. Relative ADC (rADC) of the tumors was determined as the ratio of the minimum ADC divided by the mean ADC of the contralateral unaffected white matter. $\mathrm{ADC}_{\text {min }}$ values were expressed as $\times 10^{-3}$ square millimeters per second.

For assessment of DSC-PWI data, whole-brain $\mathrm{CBV}$ maps were generated by applying a single-compartment model and an automated arterial input function. The relative maximum $\mathrm{CBV}\left(\mathrm{rCBV}_{\max }\right)$ was calculated by dividing the tumor $\mathrm{CBV}$ voxel value by the mean CBV value of the contralateral unaffected white matter to minimize variances in $\mathrm{rCBV}_{\max }$ values in each individual patient. Measurements of $\mathrm{rCBV}_{\text {max }}$ values were performed with the same ROIs as those used for ADC measurements. The ROIs for the $\mathrm{ADC}$ and $\mathrm{rCBV}$ measurements were not identical and were not from the same region of the tumor in each patient. The signal intensities on DWI, rADC, $\mathrm{ADC}_{\text {min }}$, and $\mathrm{rCBV}_{\max }$ parameters were determined by another senior neuroradiologist who was experienced in diffusion and perfusion data acquisition and blinded to the tumor histology and molecular data. This method has been shown to provide the highest interobserver and intraobserver reproducibility. ${ }^{29}$

\section{Immunohistochemistry Staining}

Immunohistochemistry was performed on $5-\mu \mathrm{m}$-thick sections from paraffin-embedded tumor specimens of all evaluated patients. Sections were incubated overnight at $4^{\circ} \mathrm{C}$ with the monoclonal antimIDH1 antibody (DIA-H09; Dianova, Hamburg, Germany) that specifically reacts with the mutant IDH1-R132, the most common glioma-derived mutation, ${ }^{30}$ but not with the wild-type IDH1. Following incubation with horseradish peroxidase-conjugated secondary antibody, the slides were then stained with the Cytomation EnVision + System horseradish peroxidase (diaminobenzidine) detection 

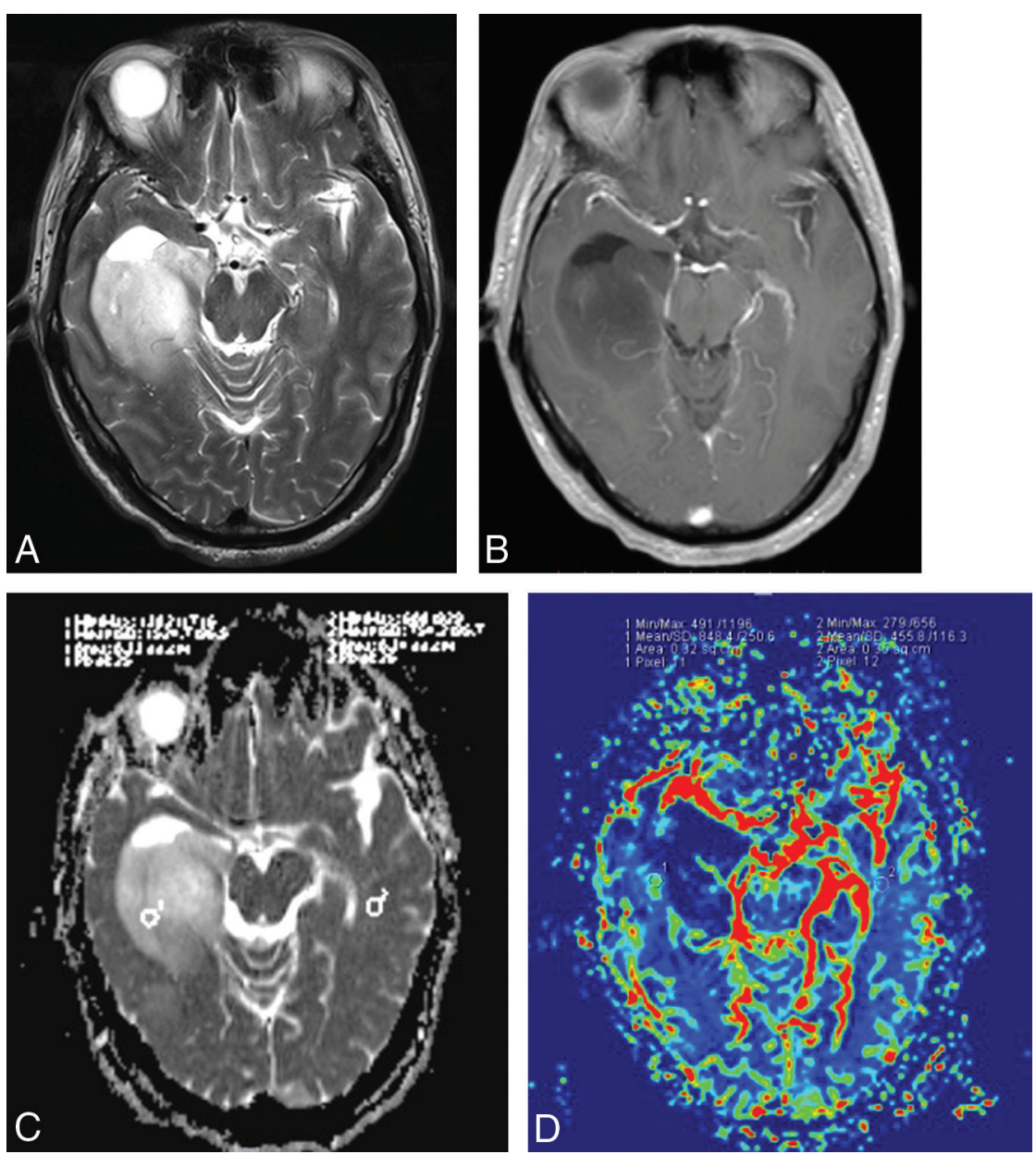

FIG 2. A 50-year-old man with an anaplastic astrocytoma with an IDH mutation. A, Axial T2WI demonstrates heterogeneous high signal intensity with sharp borders on the right temporal lobe. $B$, Contrast-enhanced axial Tl-weighted image demonstrates a nonenhancing lesion in the right temporal region. $C, A$ corresponding $A D C$ map shows the tumor with an increased $A D C$ value $\left(A D C_{\min }=1.456 \times 10^{-3} \mathrm{~mm}^{2} / \mathrm{s}, \mathrm{rADC}=2.51\right) . D$, Correlative color $\mathrm{CBV}$ image shows relatively low perfusion with the calculated $\mathrm{rCBV}_{\max }$ of 1.86 .

Table 2: Comparison of DWI and DSC-PWI variables between IDH mutation and wild-type grade II and III astrocytomas

\begin{tabular}{lccc}
\hline & IDH Mutation & IDH Wild Type & $P$ Value \\
\hline $\mathrm{ADC}_{\min }\left(\times 10^{-3} \mathrm{~mm}^{2} / \mathrm{s}\right)$ & $1.21 \pm 0.27$ & $0.87 \pm 0.18$ & $<.001$ \\
$\mathrm{rADC}$ & $1.88 \pm 0.41$ & $1.37 \pm 0.31$ & $<.001$ \\
$\mathrm{rCBV}_{\max }$ & $1.41 \pm 0.50$ & $3.47 \pm 2.34$ & .004 \\
\hline
\end{tabular}

${ }^{\text {a }}$ Data are means.

kit (Dako, Carpenteria, California) and counterstained with hematoxylin. Staining was interpreted as positive when $\geq 10 \%$ of tumor cells showed a strong cytoplasmic staining for $\mathrm{mIDH} 1$, whereas staining of $<10 \%$ of tumor cells was counted as negative findings. ${ }^{31}$

\section{DNA Sequencing}

Genomic DNA was extracted from formalin-fixed, paraffin-embedded tissue sections by using MyghtyAmp for FFPE (Takara Bio, Shiga, Japan), according to the manufacturer's instructions. Mutational alterations of $I D H 1$ and $I D H 2$ at hotspot codons R132 and R172 were assessed by a bidirectional cycle sequencing of polymerase chain reaction-amplified fragments with the following primers: IDH1f (5'-TGCCACCAACGACCAAGTCA-3') and IDH1r (5'CATGCAAAA TCACATATTTGCC-3'); IDH2f (5' -TGAAAGAT-
GGCGGCTGCAGT-3') and IDH2r (5'-GGGGTGAAGACCATTTTGAA-3').

\section{Data Analysis}

All quantitative parameters are presented as mean $\pm \mathrm{SD}$. Comparisons of $\mathrm{ADC}_{\text {min }}, \mathrm{rADC}$, and $\mathrm{rCBV}_{\max }$ values between $I D H$-mutant and wild type of grade II and III astrocytomas were made with the Mann-Whitney $U$ test. The receiver operating characteristic and logistic regression analysis were performed to determine the best cutoff value in discriminating $I D H$-mutant from wildtype tumors. The sensitivity, specificity, positive predictive value, negative predictive value, Youden index, and area under the curve based on optimum thresholds for variable parameters were calculated. We chose the cutoff value for each quantitative parameter that provided optimal sensitivity and specificity. In addition, comparisons of the areas under the curve for different variables were made with the $Z$-test. Statistical analysis was calculated in SPSS, Version 19.0 (IBM, Armonk, New York). $P<.05$ was considered significant.

\section{RESULTS}

Forty-two histologically confirmed grade II and III astrocytoma cases including 17 cases with $I D H$ mutation and 25 cases without such mutation were enrolled in this study. The clinical, histologic, and cMRI characteristics are summarized in Table 1. Twenty-five patients with no IDH1 or IDH2 mutations were older $(I D H$ mutation $=35.76 \pm 9.13$ years, $I D H$ wild type $=45.96 \pm 18.36$ years, $P=.041)$ and demonstrated more indistinct margins than those with $I D H$ mutations (IDH mutation, 6/17; IDH wild type, 17/25; $P=.037$ ). Tumors with $I D H$ mutations were more likely to occur in the frontal lobes (Figs $1 A,-B$, and $2 A,-B)$. No differences in heterogeneous appearance were observed among the groups.

The $\mathrm{ADC}_{\text {min }}$ values, $\mathrm{rADC}$ ratios, and $\mathrm{rCBV}_{\text {max }}$ calculated for $I D H$-mutant and wild-type grade II and III astrocytomas are summarized in Table 2. Both the $\mathrm{ADC}_{\min }(\mathrm{IDH}$ mutation $=1.21 \pm$ $0.27, I D H$ wild type $=0.87 \pm 0.18 ; P<.001)$ and $\operatorname{rADC}(I D H$ mutation $=1.88 \pm 0.41 ; I D H$ wild type $=1.37 \pm 0.31 ; P<.001)$ were significantly higher in $I D H$-mutant tumors than in wild types (Figs $1 C$ and $2 C$ ). The $\mathrm{rCBV}_{\max }(I D H$ mutation $=1.41 \pm$ $0.50 ; I D H$ wild type $=3.47 \pm 2.34 ; P=.004)$ in patients with $I D H$-mutant tumors was significantly lower than in those with $I D H$ wild-type tumors (Table 2 and Figs $1 D$ and $2 D)$.

The results of the receiver operating characteristic curve analysis are shown in Table 3 and Fig 3. Logistic regression analysis 
Table 3: Measurement of TV, sensitivity, specificity, PPV, NPV, YI, and AUC of ADC min $_{\text {values, }}$ ADC, rCBV $_{\max }, \mathrm{CMRI}, \mathrm{DWI}+\mathrm{DSC}-\mathrm{PWI}$, and CMRI+ DWI + DSC-PWI for assessing the IDH status of grade II and III astrocytomas

\begin{tabular}{|c|c|c|c|c|c|c|c|}
\hline & TV & Sensitivity (\%) & Specificity (\%) & PPV (\%) & NPV (\%) & $\mathrm{YI}$ & AUC $(95 \% \mathrm{CI})$ \\
\hline$A D C_{\min }$ & 1.01 & 76.92 & 82.61 & 91.20 & 60.50 & 0.60 & $0.87(0.71-0.96)$ \\
\hline rADC & 1.60 & 84.62 & 73.91 & 88.30 & 67.30 & 0.59 & $0.84(0.68-0.94)$ \\
\hline $\mathrm{rCBV}_{\max }$ & 2.35 & 100.00 & 60.87 & 85.60 & 100.00 & 0.61 & $0.82(0.66-0.93)$ \\
\hline CMRI & & 88.24 & 52.00 & 88.50 & 44.30 & 0.40 & $0.78(0.63-0.89)$ \\
\hline DWI + DSC-PWI & & 100.00 & 65.22 & 87.00 & 100.00 & 0.65 & $0.88(0.54-0.84)$ \\
\hline CMRI+ DWI + DWI-PWI & & 92.31 & 91.30 & 96.10 & 83.60 & 0.84 & $0.92(0.78-0.98)$ \\
\hline
\end{tabular}

Note:-TV indicates threshold values; PPV, positive predictive value; NPV, negative predictive value; YI, Youden Index; AUC, area under the curve.

was used to test these cMRI parameters among groups; then, a combination of cMRI, DWI, and DSC-PWI for the diagnosis of IDH mutation yielded a sensitivity, specificity, positive predictive value, negative predictive value, and Youden index of $92.31 \%$, $91.30 \%, 96.10 \%, 83.60 \%$, and 0.84 , respectively. A significant difference was found in the areas under the curve between cMRI and cMRI + DWI + DSC-PWI $(Z=2.8, P=.005)$.

\section{DISCUSSION}

The present study demonstrates that cMRI, DWI, and DSC-PWI can be used to evaluate the IDH mutational status in gliomas and that a combination of DWI, DSC-PWI, and cMRI further improves the diagnostic accuracy. We found that patients with $I D H$ wild-type tumors were significantly older than those with $I D H$ mutated tumors. This correlation between age and $I D H$ mutation status in grade II gliomas has also been reported by Metellus et al. ${ }^{17}$ Our finding that $I D H$-mutated tumors tend to reside in the frontal lobes is consistent with previous studies reporting that $I D H$-mutation tumors are strongly associated with frontal locations. ${ }^{9,28}$ In this study, all 11 tumors that did not involve the cerebral cortex were $I D H$ wild type, which confirmed a previous finding in anaplastic gliomas that tumors not located in the cerebral cortex were $I D H$-intact tumors. ${ }^{27}$ While the radiologic appearance of infiltrating lesions in anaplastic gliomas was associated with $\mathrm{IDH}$ mutation status, ${ }^{17}$ these cMRI characteristics lack the ability to quantify the findings. Indeed, it is challenging to determine molecular alterations of such tumors by using cMRI only.

$\mathrm{ADC}_{\text {min }}$ values have been extensively used to investigate brain tumors and their prognosis. ${ }^{21,32-34}$ Tan et $\mathrm{al}^{25}$ reported that $\mathrm{ADC}_{\text {min }}$ and $\mathrm{rADC}$ could be used to identify gliomas with and without IDH mutation. Similarly, Lee et $\mathrm{al}^{35}$ found that the mean ADC value was a useful parameter for differentiating IDH1 gene mutation-positive high-grade gliomas from the mutation-negative subtypes with histogram analysis. Our study demonstrated that the $\mathrm{ADC}_{\text {min }}$ and $\mathrm{rADC}$ values of $I D H$-mutant grade II and III astrocytomas were higher than those of wild types. $\mathrm{ADC}_{\min }$ has been shown to depict the sites of highest cellularity within heterogeneous tumors. $^{21,33}$ Therefore, we chose this simple-but-efficient method for the tumor analysis and differentiation. Accumulating evidence has indicated that mutation in the $I D H$ gene family could reduce catalytic generation of $\alpha$-ketoglutarate and, in turn, lead to the production of the oncometabolite (R)-2-hydroxyglutarate [(R)-2HG], ultimately giving rise to increased cell proliferation or cellularity. ${ }^{36-38}$ Therefore, it is conceivable that differences in $\mathrm{ADC}_{\text {min }}$ and $\mathrm{rADC}$ values as presented in the current study might be useful for predicting the molecular profile in astrocytomas with respect to $I D H$ mutational status.
DSC-PWI has the potential to noninvasively provide morphologic and functional information about gliomas. ${ }^{22,23}$ Law et $\mathrm{al}^{39}$ reported that DSC-PWI could be used to predict the median time to progression in gliomas and that a lower rCBV corresponds to significantly prolonged progression-free survival. However, these authors did not investigate the rCBV-related molecular mechanisms such as $I D H$ mutation. Our data suggest that $\mathrm{rCBV}_{\text {max }}$ values are significantly associated with the $I D H$ mutational status. Recent research showed that $I D H$ mutation leads to 2HG (an activator of Egl-9 prolyl-4-hydroxylases) accumulation, resulting in decreased hypoxia-inducible-factor 1- $\alpha$ activation and downstream inhibition of angiogenesis-related signaling. ${ }^{38,40}$ As demonstrated by Kickingereder et $\mathrm{al}^{18}$ that $I D H$-mutant and wild-type tumors were both associated with distinct imaging phenotypes and were predictable with $\mathrm{rCBV}$ imaging in a clinical setting, rCBV maps were used to evaluate $I D H$ mutational status of highgrade gliomas with similar results (ie, $I D H$ mutant tumors represented considerably lower rCBV). ${ }^{35}$ Similarly, the $\mathrm{rCBV}_{\max }$ values in our cases with $I D H$ mutation were significantly lower than wild types. Therefore, our findings are in good agreement with prior results and theories. In this study, $I D H$-mutated grade II and III astrocytomas were found to correlate with higher $\mathrm{ADC}_{\text {min }}$ and rADC and lower rCBV; this correlation corresponds to low levels of cellular density and angiogenesis. These relationships may explain why $I D H$ mutation is an independent favorable prognostic marker in patients with gliomas. ${ }^{14-19}$ Glioblastoma is the most common malignant and fatal type of brain tumor, with a poor prognosis. ${ }^{5,10}$ Although the presence of an $I D H$ mutation is a strong, independent prognostic factor in gliomas, it had been shown that even $I D H$-mutated glioblastomas exhibited clinical outcomes similar to those of grade III astrocytomas without IDH mutation. ${ }^{6}$ The IDH mutation is relatively rare in glioblastomas because it is associated with secondary but not primary tumors. ${ }^{5}$ Thus, glioblastomas were excluded from our study.

IDH mutation in diffuse gliomas has been considered the most robust prognostic implication in previous studies. ${ }^{10-17} \mathrm{IDH}$ mutation was associated with a significantly better clinical outcome with 5-year overall survival (93\% compared with 51\% for wild type). ${ }^{17}$ Although our results demonstrated that MR imaging parameters could noninvasively predict $I D H$ mutational status in WHO grade II and III astrocytomas, the correlation between imaging parameters and clinical outcomes has not been studied because of the retrospective nature of this study and a short-term follow-up. Nevertheless, a few studies have demonstrated that low ADC values related directly to poor survival in high-grade astrocytomas and that $\mathrm{rCBV}$ values 


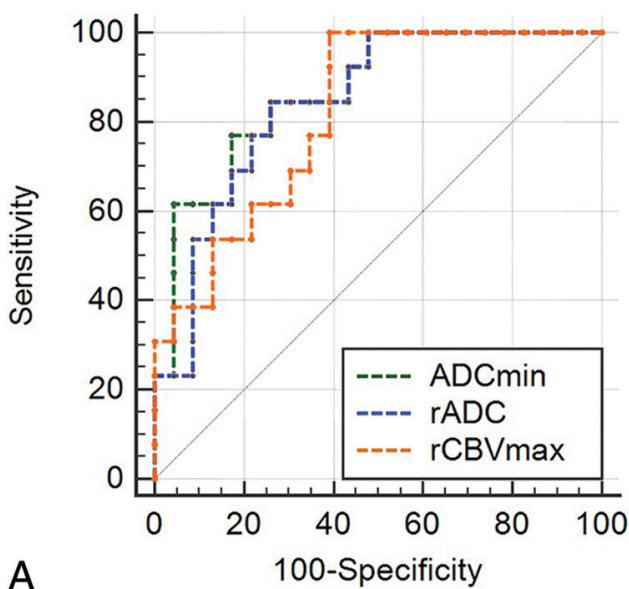

A

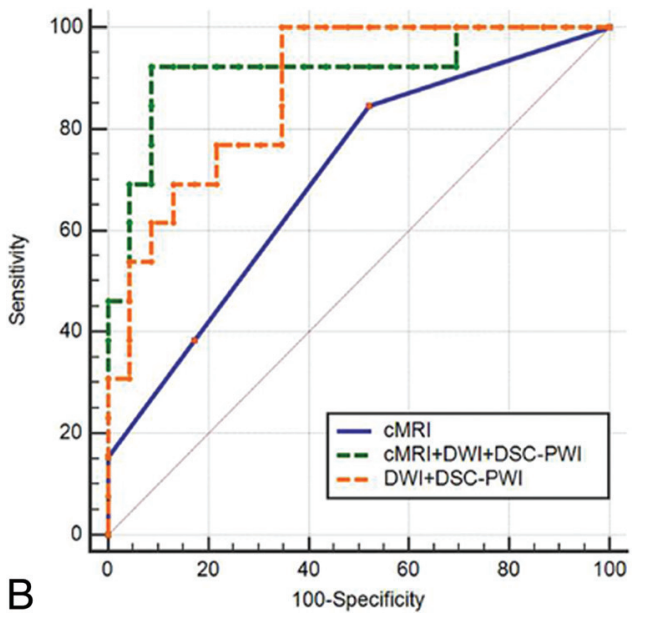

FIG 3. Comparison of receiver operating characteristic curves of $A D C_{\text {min }}, r A D C, r C B V_{\text {max }}(A)$ and $C M R I, D W I+D S C-P W I$, and $c M R I+$ $\mathrm{DWI}+\mathrm{DSC}-\mathrm{PWI}(B)$ in differentiating $\mathrm{IDH}$-mutant grade II and III astrocytomas from wild types.

were positively correlated with median time to progression in patients with gliomas. ${ }^{32,39,41,42}$

There are a few limitations to this study. It has inherent biases associated with retrospective analyses and a relatively small sample size. A multicentered prospective investigation with a larger sample size is warranted to verify these results and ensure the reproducibility. Second, owing to the short-term follow-up, the clinical outcomes are not available. Imaging parameters as predictors of clinical outcomes should be further studied in larger, prospective trials. Third, the classic biomarker of the human p53 tumor suppressor gene (TP53) mutation and O6-methylguanineDNA methyltransferase (MGMT) promoter methylation status was not used in this study for case grouping due to lack of relevant molecular pathologic data in our research. Finally, tumor borders were defined with reference to high signal intensity on T2WI and FLAIR, but it is difficult to differentiate tumor infiltration and peritumoral edema merely on the basis of their radiologic appearance.

\section{CONCLUSIONS}

Compared with $I D H$ wild-type tumors, $I D H$-mutant tumors tend to have a higher $\mathrm{ADC}_{\min }$ and $\mathrm{rADC}$ and a lower $\mathrm{rCBV}_{\max }$. Application of cMRI with advanced imaging modalities such as DWI and DSC-PWI is useful to predict $I D H$ mutational status in grade II and III astrocytomas and may provide an important, noninvasive, surrogate marker that should be studied further and clinically correlated in larger, prospective trials.

\section{REFERENCES}

1. Daumas-Duport C, Scheithauer B, O'Fallon J, et al. Grading of astrocytomas: a simple and reproducible method. Cancer 1988;62: 2152-65 Medline

2. Fuller GN, Scheithauer BW. The 2007 Revised World Health Organization (WHO) Classification of Tumours of the Central Nervous System: newly codified entities. Brain Pathol 2007;17:304-07 CrossRef Medline

3. van den Bent MJ. Interobserver variation of the histopathological diagnosis in clinical trials on glioma: a clinician's perspective. Acta Neuropathol 2010;120:297-304 CrossRef Medline

4. Louis DN, Perry A, Reifenberger G, et al. The 2016 World Health Organization Classification of Tumors of the Central Nervous System: a summary. Acta Neuropathol 2016;131:803-20 CrossRef Medline

5. Parsons DW, Jones S, Zhang X, et al. An integrated genomic analysis of human glioblastoma multiforme. Science 2008;321:1807-12 CrossRef Medline

6. Hartmann C, Meyer J, Balss J, et al. Type and frequency of IDH1 and IDH2 mutations are related to astrocytic and oligodendroglial differentiation and age: a study of 1,010 diffuse gliomas. Acta Neuropathol 2009;118:469-74 CrossRef Medline

7. Yan H, Parsons DW, Jin G, et al. IDH1 and IDH2 mutations in gliomas. N Engl J Med 2009;360:765-73 CrossRef Medline

8. Balss J, Meyer J, Mueller W, et al. Analysis of the IDH1 codon 132 mutation in brain tumors. Acta Neuropathol 2008;116:597-602 CrossRef Medline

9. Watanabe T, Nobusawa S, Kleihues P, et al. IDH1 mutations are early events in the development of astrocytomas and oligodendrogliomas. Am J Pathol 2009;174:1149-53 CrossRef Medline

10. Olar A, Wani KM, Alfaro-Munoz KD, et al. IDH mutation status and role of WHO grade and mitotic index in overall survival in grade II-III diffuse gliomas. Acta Neuropathol 2015;129:585-96 CrossRef Medline

11. Nikiforova MN, Hamilton RL. Molecular diagnostics of gliomas. Arch Pathol Lab Med 2011;135:558-68 CrossRef Medline

12. Reuss DE, Mamatjan Y, Schrimpf D, et al. IDH mutant diffuse and anaplastic astrocytomas have similar age at presentation and little difference in survival: a grading problem for WHO. Acta Neuropathol 2015;129:867-73 CrossRef Medline

13. Sanson M, Marie $\mathrm{Y}$, Paris $\mathrm{S}$, et al. Isocitrate dehydrogenase $\mathbf{1}$ codon 132 mutation is an important prognostic biomarker in gliomas. J Clin Oncol 2009;27:4150-54 CrossRef Medline

14. Bleeker FE, Atai NA, Lamba S, et al. The prognostic IDH1 (R132) mutation is associated with reduced NADP+-dependent IDH activity in glioblastoma. Acta Neuropathol 2010;119:487-94 CrossRef Medline

15. Krell D, Mulholland P, Frampton AE, et al. IDH mutations in tumorigenesis and their potential role as novel therapeutic targets. Future Oncol 2013;9:1923-35 CrossRef Medline

16. Rohle D, Popovici-Muller J, Palaskas N, et al. An inhibitor of mutant IDH1 delays growth and promotes differentiation of glioma cells. Science 2013;340:626-30 CrossRef Medline

17. Metellus P, Coulibaly B, Colin C, et al. Absence of IDH mutation identifies a novel radiologic and molecular subtype of WHO grade II gliomas with dismal prognosis. Acta Neuropathol 2010;120: 719-29 CrossRef Medline

18. Kickingereder P, Sahm F, Radbruch A, et al. IDH mutation status is associated with a distinct hypoxia/angiogenesis transcriptome signature which is non-invasively predictable with rCBV imaging in human glioma. Sci Rep 2015;5:16238 CrossRef Medline 
19. Schaefer PW, Grant PE, Gonzalez RG. Diffusion-weighted MR imaging of the brain. Radiology 2000;217:331-45 CrossRef Medline

20. Le Bihan D. Looking into the functional architecture of the brain with diffusion MRI. Nat Rev Neurosci 2003;4:469-80 CrossRef Medline

21. Humphries PD, Sebire NJ, Siegel MJ, et al. Tumors in pediatric patients at diffusion-weighted MR imaging: apparent diffusion coefficient and tumor cellularity. Radiology 2007;245:848-54 CrossRef Medline

22. Guzmán-De-Villoria JA, Mateos-Pérez JM, Fernández-García P, et al. Added value of advanced over conventional magnetic resonance imaging in grading gliomas and other primary brain tumors. Cancer Imaging 2014;14:1-10 CrossRef Medline

23. Cuccarini V, Erbetta A, Farinotti M, et al. Advanced MRI may complement histological diagnosis of lower grade gliomas and help in predicting survival. J Neurooncol 2016;126:279-88 CrossRef Medline

24. Jain R, Gutierrez J, Narang J, et al. In vivo correlation of tumor blood volume and permeability with histologic and molecular angiogenic markers in gliomas. AJNR Am J Neuroradiol 2011;32:388-94 CrossRef Medline

25. Tan WL, Huang WY, Yin B, et al. Can diffusion tensor imaging noninvasively detect IDH1 gene mutations in astrogliomas? A retrospective study of 112 cases. AJNR Am J Neuroradiol 2014;35: 920-27 CrossRef Medline

26. Khayal IS, Vandenberg SR, Smith KJ, et al. MRI apparent diffusion coefficient reflects histopathologic subtype, axonal disruption, and tumor fraction in diffuse-type grade II gliomas. Neuro Oncol 2011; 13:1192-201 CrossRef Medline

27. Sonoda Y, Shibahara I, Kawaguchi T, et al. Association between molecular alterations and tumor location and MRI characteristics in anaplastic gliomas. Brain Tumor Pathol 2015;32:99-104 CrossRef Medline

28. Carrillo JA, Lai A, Nghiemphu PL, et al. Relationship between tumor enhancement, edema, IDH1 mutational status, MGMT promoter methylation, and survival in glioblastoma. AJNR Am J Neuroradiol 2012;33:1349-55 CrossRef Medline

29. Wetzel SG, Cha S, Johnson G, et al. Relative cerebral blood volume measurements in intracranial mass lesions: interobserver and intraobserver reproducibility study. Radiology 2002;224:797-803 CrossRef Medline

30. Kato Y, Jin G, Kuan CT, et al. A monoclonal antibody IMab-1 specifically recognizes IDH1R132H, the most common glioma-derived mutation. Biochem Biophys Res Commun 2009;390:547-51 CrossRef Medline
31. Takano S, Tian W, Matsuda M, et al. Detection of IDH1 mutation in human gliomas: comparison of immunohistochemistry and sequencing. Brain Tumor Pathol 2011;28:115-23 CrossRef Medline

32. Higano S, Yun X, Kumabe T, et al. Malignant astrocytic tumors: clinical importance of apparent diffusion coefficient in prediction of grade and prognosis. Radiology 2006;241:839-46 CrossRef Medline

33. Murakami R, Hirai T, Sugahara T, et al. Grading astrocytic tumors by using apparent diffusion coefficient parameters: superiority of a one- versus two-parameter pilot method. Radiology 2009;251: 838-45 CrossRef Medline

34. Lee EJ, Lee SK, Agid R, et al. Preoperative grading of presumptive low-grade astrocytomas on MR imaging: diagnostic value of minimum apparent diffusion coefficient. AJNR Am J Neuroradiol 2008; 29:1872-77 CrossRef Medline

35. Lee S, Choi SH, Ryoo I, et al. Evaluation of the microenvironmental heterogeneity in high-grade gliomas with IDH1/2 gene mutation using histogram analysis of diffusion-weighted imaging and dynamic-susceptibility contrast perfusion imaging. J Neurooncol 2015;121:141-50 CrossRef Medline

36. Dang L, White DW, Gross S, et al. Cancer-associated IDH1 mutations produce 2-hydroxyglutarate. Nature 2009;462:739-44 CrossRef Medline

37. Xiong J, Tan W, Wen J, et al. Combination of diffusion tensor imaging and conventional MRI correlates with isocitrate dehydrogenase $1 / 2$ mutations but not $1 \mathrm{p} / 19 \mathrm{q}$ genotyping in oligodendroglial tumours. Eur Radiol 2016;26:1705-15 CrossRef Medline

38. Losman JA, Looper R, Koivunen P, et al. (R)-2-hydroxyglutarate is sufficient to promote leukemogenesis and its effects are reversible. Science 2013;339:1621-25 CrossRef Medline

39. Law M, Young RJ, Babb JS, et al. Gliomas: predicting time to progression or survival with cerebral blood volume measurements at dynamic susceptibility-weighted contrast-enhanced perfusion MR imaging. Radiology 2008;247:490-98 CrossRef Medline

40. Koivunen P, Lee S, Duncan CG, et al. Transformation by the (R)enantiomer of 2-hydroxyglutarate linked to EGLN activation. $\mathrm{Na}$ ture 2012;483:484-88 CrossRef Medline

41. Mangla R, Ginat DT, Kamalian S, et al. Correlation between progression free survival and dynamic susceptibility contrast MRI perfusion in WHO grade III glioma subtypes. J Neurooncol 2014;116: 325-31 CrossRef Medline

42. Zulfiqar M, Yousem DM, Lai H. ADC values and prognosis of malignant astrocytomas: does lower ADC predict a worse prognosis independent of grade of tumor? A meta-analysis. AJR Am J Roentgenol 2013;200:624-29 CrossRef Medline 